\title{
Dextrose, Maltodextrina e Waxy Maize: principais diferenças na composição, mecanismo de ação e recomendações para o desempenho esportivo
}

\author{
Dextrose, Maltodextrin and Waxy Maize: main differences in \\ composition, mechanism of action and recommendations for sportive \\ performance
}

\author{
1 Mariana Cardoso \\ 2 Thais Tavares Pereira Seabra \\ 1 Elton Bicalho de Souza elton.bicalho01@gmail.com \\ $\begin{array}{ll}1 & \text { Centro Universitário de Volta Redonda } \\ 2 & \text { UNIVERSO - Universidade Salgado de Oliveira }\end{array}$
}

\section{RESUMO}

Os suplementos energéticos para atletas têm como principal ingrediente o carboidrato, principal fornecedor de energia para o organismo. Dentre os diferentes tipos de suplementos energéticos, destacam-se a dextrose, a maltodextrina e o waxy maize, cada um uma característica distinta em sua composição e função. Essas diferenças geram dúvidas no momento da escolha, visto que cada um possui um tipo de resposta glicêmica. Em virtude disso, o presente estudo teve como objetivo descrever, de acordo com a literatura, as principais diferenças entre esses três suplementos, demonstrando em qual momento seria mais indicado a utilização de cada um. Trata-se de um estudo de revisão de literatura, no qual o levantamento dos dados foi realizado em livros da área de Nutrição, Fisiologia e Bioquímica, bem como artigos publicados nos últimos 10 anos nos portais Google Acadêmico ${ }^{\circledR}$ e Scielo ${ }^{\circledR}$. Foi possível concluir que a dextrose e a maltodextrina, apesar de serem tipos distintos de carboidratos, possuem respostas glicêmicas semelhantes e, em contrapartida, o waxy maize possui uma resposta glicêmica mais lenta.

\section{Palavras-chave}

Suplementos nutricionais; carboidrato; desempenho atlético.

\section{ABSTRACT}

The energy supplements for athletes have as the main ingredient the carbohydrate, which is the main energy provider for the human organism. Among the different kinds of energy supplement, dextrose, maltodextrin and waxy maize are highlighted, and each one has a distinct characteristic in its composition and function. These differences beget doubts during the moment of choice, as each one possesses a type of glycemic answer. Because of this fact, the present study had as its objective to describe, according to the literature, the main differences among these three supplements, demonstrating in which moment it would be more indicated the utilization of them. It is a literature review study, in which the data collecting was done through Nutrition, Physiology and Biochemistry books, as well as articles published in the last 10 years on Google Academic $^{\circledR}$ and Scielo ${ }^{\circledR}$. It was possible to conclude that dextrose and maltodextrin, though being distinct from carbohydrates, have similar glycemic answers and, on the other hand, waxy maize has a slower glycemic answer.

\section{Keywords}

Dietary Supplements; Carbohydrate; Athletic performance.

\section{Como você deve citar?}

CARDOSO, Mariana; SOUZA, SEABRA, Thais Tavares Pereira; Elton Bicalho de; Dextrose, Maltodextrina e Waxy Maize: principais diferenças na composição, mecanismo de ação e recomendações para o desempenho esportivo. Cadernos UniFOA, Volta Redonda, n. 33, p. 101-109, abr. 2017. 
Dextrose, Maltodextrina e Waxy Maize: principais diferenças na composição, mecanismo

de ação e recomendações para o desempenho esportivo

\section{INTRODUÇÃO}

Historicamente, nota-se o aumento do consumo de ergogênicos nutricionais (EN), popularmente conhecidos como suplementos nutricionais que, de acordo com a Resolução CFN n 390/2006 e com o Dietay Supplement Health Education Act (DSHEA), é um produto alimentar que, acrescido à dieta, contém pelo menos um dos seguintes ingredientes: vitaminas, minerais, aminoácidos, ácidos graxos, carboidratos, todavia o DSHEA ainda complementa sua definição com plantas ou ervas, metabólito, constituinte ou extrato ou a combinação desses ingredientes supracitados. De acordo com a Resolução n 18 da Agência Nacional de Vigilância Sanitária - ANVISA, esses suplementos são divididos em: suplemento hidroeletrolítico para atletas; suplemento proteico para atletas; suplemento de creatina para atletas e; suplemento energético para atletas, escopo do presente trabalho (DSHEA, 1994; BRASIL, 2006; BRASIL, 2010).

Os suplementos energéticos são produtos destinados a complementar as necessidades energéticas do atleta, devendo atender aos seguintes requisitos: conter um mínimo de $75 \%$ do valor energético total (VET) proveniente de carboidratos (CHO); conter, no mínimo, 15 gramas de $\mathrm{CHO}$ na porção pronta do produto; ser adicionado de vitaminas e/ou minerais; conter proteínas intactas e/ou parcialmente hidrolisadas; lipídeos e; não ser adicionado de fibras alimentares e não nutrientes (BRASIL, 2010). Nota-se que o $\mathrm{CHO}$ é o principal ingrediente desse tipo de suplemento. Trata-se de um macronutriente composto basicamente por carbono, hidrogênio e oxigênio, sendo sua fórmula geral: $\mathrm{CH}_{2} \mathrm{O}$. Possui a importante função de ser o principal fornecedor de energia ao organismo, provendo cerca de metade do total de calorias da dieta. Pode ser classificado em: monossacarídeos, dissacarídeos, oligossacarídeos e polissacarídeos, de acordo com o tamanho de sua molécula. Em decorrência disso e por ser o principal substrato para o desempenho físico, a utilização de fontes de $\mathrm{CHO}$ na prática esportiva é indispensável, visto que, normalmente, há um elevado gasto energético (GE) decorrente de determinadas atividades (SHILS et al., 2003; MAHAN et al., 2012; BERG; TYMOCZKO; STRYER, 2014; LONGO, 2014).

Atualmente, observa-se o consumo dos suplementos dextrose (DEX), maltodextrina (MAL) e Waxy Maize (WM) por desportistas. A DEX constitui o tipo mais conhecido e mais simples de $\mathrm{CHO}$, o monossacarídeo. A MAL é um tipo de amido hidrolisado, ou seja, é um oligossacarídeo de fácil absorção, normalmente utilizada em bebidas esportivas. Já o WM é um tipo de amido de milho ceroso (Waxy Maize Starch), ou seja, é um polissacarídeo, proveniente das variedades de milho de baixa osmolaridade, cevada e arroz, que criam cadeias ramificadas de amilopectina (HENRIQUES et al., 2010; MAHAN et al., 2012).

A principal diferença entre esses três suplementos é o índice glicêmico de cada um, normalmente utilizado para classificar diferentes tipos de $\mathrm{CHO}$, de acordo com a sua capacidade de elevar a resposta glicêmica, que é uma característica utilizada no momento da escolha, por ser uma estratégia que permite ao atleta a manutenção das taxas de glicose mais elevadas durante o exercício, evitando a liberação exacerbada de insulina e mantendo as reservas de glicogênio por mais tempo (PEREIRA, 2007; MAHAN et al., 2012). Diante do exposto, questiona-se: qual desses suplementos possui melhor efeito para o desportista? Como realizar a escolha para diferentes tipos de atividades?

Em face do exposto, o objetivo do presente estudo foi descrever, segundo a literatura científica, as principais diferenças entre dextrose, maltodextrina e waxy maize, no que tange à composição, mecanismo de ação e performance esportiva. 


\section{MÉTODOS}

Trata-se de uma revisão da literatura científica em que foram utilizados como instrumentos de pesquisa livros técnicos da área de Nutrição, Fisiologia e Bioquímica, bem como artigos publicados nos últimos 10 anos nos portais Google Acadêmico ${ }^{\circledast}$ e Scielo ${ }^{\circledast}$. Foram pesquisadas obras publicadas em português e inglês, utilizando-se os seguintes termos de busca: "maltodextrina" or "maltodextrin", "dextrose" or "dextrhose", "amido ceroso de milho" or "waxy maize" e "fadiga muscular" or "muscle fatigue".

\section{DEXTROSE}

A DEX constitui o tipo mais conhecido e mais simples de $\mathrm{CHO}$, os monossacarídeos. São carboidratos fundamentais para outros tipos de $\mathrm{CHO}$, como a maltodextrina, a maltose, entre outros, pois compõem a estrutura química deles, além de ser uma das principais fontes de energia em praticamente todas as formas de vida (BERG; TYMOCZKO; STRYER, 2014; VOET; VOET; PRATT, 2014).

Os monossacarídeos são moléculas orgânicas que, de acordo com a localização da carbonila, podem ser nomeados de aldeídos ou cetonas. Geralmente, são derivados de poli-hidroxiálcoois de cadeia linear, contendo pelo menos três átomos de carbono. A DEX possui um aldeído no seu grupo carbonila, baixo peso molecular e é composta por seis átomos de carbono, doze de hidrogênio e seis de oxigênio $\left(\mathrm{C}_{6} \mathrm{H}_{12} \mathrm{O}_{6}\right)$. Dessa forma, pode-se classificá-la como uma Aldo-hexose $\mathrm{D}$-glicose. $\mathrm{O}$ seu grupo carbonila encontra-se no início da cadeia carbônica. É facilmente encontrada na natureza, podendo ser produzida a partir da gliconeogênese, através da ingestão de $\mathrm{CHO}$ complexos, ou podem ser sintetizados a partir de precursores menores, derivados de gás carbônico $\left(\mathrm{CO}_{2}\right)$ e água $\left(\mathrm{H}_{2} \mathrm{O}\right)$, pela fotossíntese. Devido ao grau de polimerização, a DEX possui uma rápida resposta glicêmica (MAHAN et al., 2012; VOET; VOET, 2013; BERG; TYMOCZKO; STRYER, 2014; LONGO, 2014; VOET; VOET; PRATT, 2014; FONTAN; AMADIO, 2015).

A absorção da DEX ocorre de duas maneiras: difusão facilitada e cotransporte de sódio e glicose. Esses processos permitem a passagem da DEX através das membranas celulares ricas em lipídios. 0 cotransportador de sódio e glicose é o Sodium glucose transporter 1 (SGLT1), que está presente nas células epiteliais absortivas da membrana apical. Esse cotransportador é responsável por transportar a DEX e a galactose, concomitantemente, a quantidades equimolares de sódio. Esse transporte ocorre por difusão facilitada, de forma a não gastar moléculas de Adenosina Tri-Fosfato (ATP). Porém, de uma forma indireta, há gasto de energia, visto que, para o sódio ser liberado no capilar (membrana basolateral), faz-se necessária a utilização da bomba sódio-potássio dependente da enzima adenosina trifosfatase (ATPase) encontrada no capilar. A partir daí, a DEX passa a ser transportada através da membrana basolateral por difusão facilitada (CHEMIN; MURA, 2010; RIBEIRO et al., 2011).

A difusão facilitada ocorre com o auxilio de uma família de transportadores conhecida como transportadores de glicose (GLUT - glucose transporters). Os GLUT são proteínas de membrana encontradas em todas as células, e são capazes de transportar a glicose a favor de seu gradiente de concentração. O transportador GLUT4 é um transportador de glicose sensível à ação da insulina. Dessa forma, está associado ao músculo esquelético, visto que ele é um tecido sensível à insulina. Em razão disso, Rodnick et al. (1992 apud Ribeiro et al., 2011) descrevem que o GLUT4 é o transportador que, durante a prática do exercício físico, apresenta maior atividade. Isso se deve ao aumento intracelular de cálcio ou a maior síntese de óxido nítrico promovidas pelo exercício físico. Portanto o GLUT4 pode ser considerado o responsável pela captação da glicose sanguínea para dentro dos músculos. Logo, durante o exercício físico, as proteínas que são armazenadas nas vesículas intracelulares são ativadas e, por conta disso, o GLUT 4 se translocará para a membrana sarcoplasmática, permitindo a entrada da glicose nas células musculares, por meio da difusão facilitada (CHEMIN; MURA, 2010; RODNICK et al., 1992 apud RIBEIRO et al., 2011). 
Dextrose, Maltodextrina e Waxy Maize: principais diferenças na composição, mecanismo de ação e recomendações para o desempenho esportivo

Dentro das células musculares, a DEX sofre processos oxidativos, como a glicólise anaeróbica ou metabolismo anaeróbico lático, que é o sistema de obtenção de energia predominante nos primeiros 45 a 90 segundos de exercício físico, propiciando uma liberação energética mais rápida do que o metabolismo aeróbico. Nesse processo, a DEX passa por reações mediadas por enzimas específicas que tem como resultado final duas moléculas de piruvato. 0 piruvato, como resultado da glicólise anaeróbica, transforma-se em ácido láctico (lactato) e, quando encontra-se aumentado na corrente sanguínea, apresenta relação direta com o declínio da força e performance. Esse fato deve-se ao desequilíbrio das reações químicas que ocorrem nos miócitos, resultando na fadiga, sensação de queimação muscular e câimbras, situações comuns em exercícios de longa e intensa duração (FOX, 2007; CHEMIN; MURA, 2010; FONTAN; AMADIO, 2015).

Com a continuação do exercício, inicia-se a glicólise aeróbica ou metabolismo aeróbico, que compreende a conversão de glicose em ácido pirúvico. Nesse processo, as moléculas de piruvato deixam o citoplasma celular e entram na mitocôndria das células, passam por um processo de descarboxilização oxidativa, convertendo-se em acetil-coenzima A (Acetil-CoA) e, por sua vez, em oxalacetato. Posteriormente, será iniciado o segundo estágio do fracionamento da glicose, o ciclo de Krebs - ou ciclo do ácido cítrico, para que ocorra a formação de ATP, responsável pela produção de energia nas células (FOX, 2007; CHEMIM; MURA, 2010; FONTAN; AMADIO, 2015).

Ante o exposto, pode-se dizer que a DEX, por conter carboidratos simples em sua composição, é de considerável importância para praticantes de exercício físico, visto que os carboidratos têm como principal função prover energia para o organismo e, dessa maneira, prevenir situações de fadiga, câimbras e queimação muscular. Sua utilização é recomendada, normalmente, antes, durante e após o exercício físico. De acordo com Sapata et al. (2006), antes do exercício, a DEX tem a capacidade de promover uma reserva energética no lúmen gastrintestinal, que será utilizado no decorrer do exercício, de forma a prevenir ou retardar algumas alterações, como distúrbios homeostáticos.

Durante a prática de exercícios de longa duração, recomenda-se o consumo da DEX, para cada hora de exercício, a fim de se evitar a hipoglicemia, fadiga e depleção do glicogênio. 0 pós-exercício é um momento relevante para se consumir a DEX, pois o organismo encontra-se estimulado para a captação de glicose (janela de oportunidade), devido à contração muscular e, como a DEX é um tipo de $\mathrm{CHO}$ de alto índice glicêmico, poderá favorecer a recuperação do esforço, pelo fato de alimentos com alto índice glicêmico promoverem maior incorporação de glicose como glicogênio muscular (HIRSCHBRUCH; CARVALHO, 2008; HERNANDEZ; NAHAS, 2009; CHEMIM; MURA, 2010).

\section{MALTODEXTRINA}

A maltodextrina (MAL) constitui um tipo mais complexo de carboidrato, se comparado com a dextrose, sendo classificada como um oligossacarídeo, ou seja, um polímero de glicose, proveniente da conversão enzimática do amido de milho, formado por monossacarídeos unidos por ligações glicosídicas. A concentração dos polímeros de glicose encontrados nesse suplemento varia de 5 a $20 \%$ (FRANÇA, 2010; VOET; VOET; PRATT, 2014).

Os oligossacarídeos são formados por pequenas cadeias de monossacarídeos (3 a 10 unidades), podendo ser denominados tri a pentassacarídeos, classificação que dependerá do número de monossacarídeos presentes na molécula. As ligações glicosídicas presentes na estrutura da MAL consistem em duas unidades de D-glicose, unidas por uma ligação a 1-4, sendo sua forma molecular: $\mathrm{C}_{6 n} \mathrm{H}_{(10 n+2)}$ $\mathrm{O}_{(5 n+1)^{*}}($ KEARSLEY; DZIEDZIC, 1995; SHILS et al., 2003; VOET; VOET; PRATT, 2014). 
Diante do exposto, observa-se que os oligossacarídeos possuem uma ligação covalente de monossacarídeos, a ligação glicosídica. Nesse tipo de ligação, ocorre a remoção de uma molécula de água. Dessa forma, a ligação glicosídica é sempre acompanhada de uma letra grega (a ou $\beta$ ), que dependerá da posição da hidroxila $(-\mathrm{OH})$ do carbono $1\left(\mathrm{C}_{1}\right)$ e dos átomos de hidrogênio do primeiro monossacarídeo; e de dois números que identificam os átomos de carbono, dos quais foi removida a molécula. É importante entender esse tipo de ligação, visto que ela é necessária para compreender o processo de digestão dos carboidratos, pois as enzimas são específicas para cada tipo de ligação glicosídica, ou seja, a enzima que hidrolisa a ligação a 1-4 não hidrolisaria uma ligação $\beta$ 1-4 ou a 1-6 (CHEMIM; MURA, 2010).

Assim, o processo digestivo da MAL se inicia na cavidade oral, pela ação da amilase salivar, que digere parcialmente a amilose em maltose e a amilopectina em maltose e dextrina. A amilase salivar continuará sua ação no estomago, no interior do bolo alimentar, transformando de 30 a $40 \%$ do amido em maltase e isomaltase. Em geral, a ação da amilase salivar seria bloqueada, após o bolo alimentar se misturar por completo à secreção gástrica, porém, o produto final do amido e as proteínas e aminoácidos presentes em uma refeição mista agem como um tampão ácido no estômago, o que permite que o processo de hidrólise do amido continue. A partir desse ponto, a digestão do amido continuará no duodeno, após a secreção exócrina do pâncreas ser estimulada pela liberação de secretina e colecistoquinina (CKK), liberando a enzima a-amilase pancreática. Contudo, quando essa enzima é adicionada aos conteúdos do estômago, ela não pode hidrolisar ligações ramificadas a 1-6. Assim, o resultado final da ação dessas enzimas consiste na liberação de grandes oligossacarídeos (dextrinas, com no mínimo uma ligação a 1-6 e com terminação a), contendo cerca de oito unidades monoméricas de glicose. Esses oligossacarídeos serão hidrolisados por enzimas denominadas glicoamilases, que removerão sequencialmente uma única unidade de glicose da extremidade não reduzida, formando moléculas de maltose e isomaltose (SHILS et al., 2003; CHEMIM; MURA, 2010).

Posteriormente, a maltose e a isomaltose serão digeridas por dissacaridases específicas (maltase e isomaltase) na membrana do enterócito, tendo como resultado final moléculas de glicose livre, que seguirão o mesmo processo de absorção da dextrose (CHEMIM; MURA, 2010).

Em geral, os oligossacarídeos são conhecidos por serem resistentes à ação digestiva em humanos, porém, a maltodextrina é uma exceção, pois é considerada um carboidrato de elevado índice glicêmico, capaz de manter os mesmos níveis glicêmicos. Além disso, por ser altamente solúvel em água, a MAL é hidrolisada e absorvida com facilidade, possui baixo valor osmótico e sabor neutro (CHEMIM; MURA, 2010; HENRIQUES et al., 2010; FONTAN; AMADIO, 2015).

Sapata et al.(2006) verificaram um aumento significativo na glicemia de indivíduos saudáveis, após o consumo de solução contendo MAL, em um período de 30 minutos após a ingestão. Além disso, Mamus et al. (2006) relataram que um dos benefícios da MAL é a manutenção dos níveis de glicose sanguínea, evitando, dessa forma, o decréscimo no desempenho durante o exercício associado à hipoglicemia. Por essa razão e por evitar a depleção de glicogênio em exercícios aeróbicos intensos, adiando a fadiga muscular provocada por esses tipos de exercícios, e por auxiliar no aumento dos estoques de glicogênio muscular, a MAL é um suplemento interessante a ser utilizado por atletas antes, durante e após o exercício físico, com o intuito de repor as reservas de glicogênio muscular, principalmente em exercícios prolongados, visto que, à medida que intensidade dos exercícios aumenta, a utilização de $\mathrm{CHO}$ torna-se cada vez mais importante para suprir a demanda do organismo (SHILS et al., 2003; FRANÇA, 2010). 
Dextrose, Maltodextrina e Waxy Maize: principais diferenças na composição, mecanismo

de ação e recomendações para o desempenho esportivo

\section{WAXY MAIZE}

O waxy maize (WM) é proveniente das variedades de milho de baixa osmolaridade, o amido de milho ceroso (Waxy Maize Starch), que cria cadeias ramificadas de amilopectina. Encontra-se na classe dos polissacarídeos, que são polímeros de médio a alto peso molecular que diferem uns dos outros na identidade das unidades de monossacarídeos repetidas, no comprimento das cadeias, nos tipos de ligações e no grau de ramificação (NELSON; COX, 2011; MAHAN, et. al., 2012).

De acordo com as taxas de glicose liberadas a partir do amido e da velocidade com que é absorvida, é possível classificá-los em três categorias distintas: o amido rapidamente digerível, o amido resistente e o amido lentamente digerido - que inclui o WM, proveniente do amido de milho ceroso (SANDS et al., 2009).

O amido, em geral, é uma mistura de glicanos, ou seja, é um homopolissacarídeo composto por macromoléculas de amilopectina (insolúvel na água) e a-amilose (hidrossolúvel), que são depositados nos cloroplastos das células vegetais. A amilopectina é considerada uma das maiores moléculas presentes na natureza, pois contém até $10^{6}$ de resíduos de glicose, unidas por ligações a (1-4). Entretanto, por ser uma molécula altamente ramificada, é possível observar que nos pontos de ramificação que ocorrem a cada 24 a 30 resíduos, as ligações são do tipo a (1-6). Além disso, a amilopectina é formada por cadeias curtas com aproximadamente 15 unidades de glicose, o que reduz sua habilidade de formar emaranhados. Em contrapartida, a a-amilose é um isômero da celulose, ou seja, consiste de cadeias longas formadas por 40 a 60 unidades de glicose, não ramificadas, unidas por ligações a (1-4), o que propicia a formação de emaranhados (CORRADINI et al., 2005; WEBER; COLLARES-QUEIROZ; CHANG, 2009; NELSON; COX, 2011; VOET; VOET; PRATT, 2014).

Poucos são os estudos envolvendo esse tipo de amido, porém Weber, Collares-Queiroz e Chang (2009) relataram que o teor de amilose nos grânulos de amido geralmente encontra-se entre $20-30 \%$ em amidos normais de cereais, mas pode variar de acordo com a fonte vegetal de origem. 0 amido de milho normalmente contém entre $25-28 \%$ de amilose, mas algumas variedades desse amido, referidas pelo termo ceroso (waxy), são constituídas quase que totalmente por amilopectina. Além disso, por ser um tipo de amido lentamente digerível, o WM proporciona uma menor resposta glicêmica e, consequentemente, uma menor resposta insulínica (PEREIRA, 2007).

Ante o exposto, observa-se que, por ser um tipo de amido, o WM possui ligações glicosídicas assim como a MAL. Por essa razão, o processo digestivo do WM ocorrerá da mesma maneira que o processo digestivo da MAL. Além disso, é importante relatar que os polissacarídeos normalmente são degradados a dissacarídeos e a monossacarídeos no trato gastrointestinal que, posteriormente serão absorvidos no intestino delgado. Porém, como o WM é composto quase totalmente por amilopectina, é importante citar como ocorre sua digestão. A amilopectina será degradada pela ação de uma $\beta$-amilase em seus pontos de ligação a (1-4), que terá como resultado as dextrinas $\beta$-limite, que são cadeias residuais que contêm os pontos de ramificação. Posteriormente, sofrerá a ação das enzimas pululanase e isoamilase, que atuam nas ligações a (1-6), produzindo a maltose. Essa maltose será digerida pela maltase e terá como produto final a glicose e, a partir daí, seguirá o mesmo processo de absorção da DEX (MENEZES; LAJOLO, 2006; WAITZBERG, 2009; CHEMIM; MURA, 2010).

Zhang, Venkatachalam e Hamaker (2006) relataram que o WM é digerido enzimaticamente in vitro entre 20 a 120 minutos e, com base nesse estudo, concluíram que, em uma refeição contendo WM, as concentrações de glicose e insulina foram mais baixas, quando comparada a uma refeição contendo amido de rápida absorção. Além disso, Thomas et al. (2006) relataram que a ingestão de $35 \mathrm{~g}$ de $\mathrm{CHO}$ 
provenientes do amido de milho ceroso resultou num aumento pequeno e sustentado da glicose no plasma, em comparação com a maltodextrina.

Levando-se em consideração o tempo de liberação de glicose e insulina no organismo decorrente do consumo do WM, ele pode ser uma alternativa muito válida, caso seja do interesse no atleta obter uma liberação mais lenta de carboidrato, para prolongar a liberação de energia pelo organismo. Dessa maneira, o WM seria capaz de evitar a hipoglicemia durante a prática do exercício físico e propiciar maior desempenho ao atleta (SANDS et al., 2009).

\section{CONSIDERAÇÕES FINAIS}

De acordo com os pontos abordados na presente revisão, nota-se que a dextrose e a maltodextrina diferem-se no tipo de carboidrato utilizado em sua composição, sendo a maltodextrina um tipo mais complexo de carboidrato, quando comparado à dextrose. Porém, apesar de ser um carboidrato mais complexo, a maltodextrina apresenta um índice glicêmico diferente dos demais carboidratos complexos, uma vez que é hidrolisada e absorvida facilmente pelo organismo. Portanto, ambos são semelhantes, quando se trata de resposta glicêmica. Logo, o efeito da utilização da dextrose nos momentos de pré, intra e pós-treino será semelhante ao efeito da maltodextrina.

Diferentemente, o waxy maize é um tipo de carboidrato mais complexo, de difícil hidrólise e absorção. Dessa forma, a resposta glicêmica desse produto será diferente dos mencionados acima. Assim o consumidor deverá atentar-se para o uso desse carboidrato, visto que ele deverá ser usado em situações em que se deseja uma liberação gradual de energia durante o exercício.

Embora mostradas algumas diferenças entre os três tipos de suplementos energéticos, observou-se que são escassos os estudos envolvendo a suplementação com wazy maize, além de não haver pesquisas comparando esses três tipos de carboidratos. Portanto, devido ao elevado consumo desses suplementos, ressalta-se a necessidade de estudos com o intuito de comparar esses diferentes suplementos, a fim de evidenciar se as diferenças relatadas pela literatura científica mostram-se evidentes na prática.

\section{REFERÊNCIAS}

BERG, J.M; TYMOCZKO, J.L; STRYER, L. Bioquímica. 7 ed. Guanabara koogan, 2014. p. 321-330.

BRASIL. Agência Nacional de Vigilância Sanitária - ANVISA. Resolução CFN nº 390. Regulamenta a Prescrição Dietética de Suplementos Nutricionais pelo Nutricionista e dá outras providências. Diário Oficial da União, Brasília, 27 de Outubro de 2006.

BRASIL. Agência Nacional de Vigilância Sanitária - ANVISA. Resolução RDC nº 18. Regulamento Técnico sobre Alimentos para Atletas. Diário Oficial da União, Brasília, 27 de Abril de 2010.

CORRADINI, E; LOTTI, C; MEDEIROS, E.S; CARVALHO, A.J.F; CURVELO, A.A.S; MATTOSO, L.H.C. Estudo comparativo de amidos termoplásticos derivados do milho com diferentes teores de amilose. Ciência e tecnologia, v. 15, n 4, p. 168-173, 2005.

COUTINHO, V.F; MENDES, R.R; ROGERO, M.M. Bioquímica e metabolismo dos carboidratos. In: CHEMIN S.M.S.S; MURA S.D.P. Tratado de alimentação, nutrição e dietoterapia. São Paulo: Roca, 2010. p. $23-41$. 
Dextrose, Maltodextrina e Waxy Maize: principais diferenças na composição, mecanismo

de ação e recomendações para o desempenho esportivo

NACIONAL INSTITUTES OF HEALTH. Office of Dietary Supplements. Dietary Supplements Health and Education Act. Public Low. US Government, 1994, p. 103-417.

HERNANDEZ A.J; NAHAS R.M. Modificações dietéticas, reposição hídrica, suplementos alimentares e drogas: comprovação de ação ergogênica e potenciais de riscos para a saúde. Sociedade Brasileira de Medicina do Esporte. v. 15, n. 3, p. 4-5, 2009.

KENNEDY J.F; KNILL C.J; TAYLOR D.W, Maltodextrins. In: DIEDZIC S.Z; KERASLEY M.W. Handbook of Starch Hydrolysis Products and their Derivatives. p.65-82, Blackie Academic \& Professional, London, 1995.

FONTAN, J.S; AMADIO, M.B. O uso de carboidratos antes da atividade física como recurso ergogênico: revisão sistemática. Revista Brasileira de Medicina do Esporte. v. 21, n. 2, p. 154-156, 2015.

FOX, S.I. Fisiologia humana. 7 ed. São Paulo: Manole, 2007.

FRANÇA, V.F. Efeito da suplementação com cafeína e maltodextrina no desempenho físico agudo de ratos wistar. Tese (mestrado). Universidade Federal do Paraná. Curitiba, 2010.

GALLAGHER M.L. Ingestão: os nutrientes e seu metabolismo. In: MAHAN, L.K; ESCOTT - STUMP, S.; RAYMOND, J.L. Alimentos, Nutrição e Dietoterapia. 13. ed. Rio de Janeiro: Elsevier, 2012. p. 33-39.

HENRIQUES, G.S; LOPÉS, C.V.A; SIMEONE, M.L.F; BINDA, C.C. Aplicação do método de índice glicêmico em modelo de ratos: padronização utilizando fórmula enteral de composição definida para animais normais e feitos diabéticos por aplicação de estreptozotocina. Revista do Médico Residente. v. 12, n. 2, p. 67-8. 2010.

HISCHBRUCH, M.D; CARVALHO, J.R. Nutrição esportiva. 2 ed. São Paulo: Manole, 2008.

LEVIN R.J, Carboidratos. In: SHILS M.E; OLSON J.A; SHIKE M; ROSS A.C. Tratado de nutrição moderna na saúde e na doença. 9. ed., v.1. São Paulo: Manole, 2003. p. 55-69.

MAMUS, R.T; SANTOS, M.G; CAMPBELL, B; KREIDER, R. Biochemical effects of carbohydrate supplementation in a simulated competition of short terrestrial duathlon. Journal of the International Society of Sports Nutrition, v. 3, n. 6, p. 11, 2006.

MENEZES, E.W; LAJOLO, F.M. Carbohidratos em alimentos regionales Iberoamericanos. Revista Brasileira de Ciências Farmacêuticas. v. 42, n. 4, p. 309-312. São Paulo, 2006.

NELSON, D.L; COX, M.M. Princípios de bioquímica de Legninger. 5 ed. Porto Alegre: Artmed, 2011.

PADILHA H.G; RODRIGUES T, Carboidratos. In: LONGO S. Manual de nutrição para o exercício físico. São Paulo: Atheneu, 2014. p. 73-75.

PEREIRA, K.D. Amido resistente, a última geração no controle de energia e digestão saudável. Ciência e Tecnologia de Alimentos. v. 27, p. 88-91. Campinas, 2007.

RODNICK, K.J et al. Immunocytochemical and biochemical studies of GLUT 4 in rat skeletal muscle. 1992. In: RIBEIRO, H.Q.T; CAMARGO, R.G; LIMA, W.P; ZANUTO, R; JUNIOR, L.C.C. Adaptações agudas promovidas por exercícios no aumento gênico, conteúdo e translocação da proteína GLUT-4 no músculo 
esquelético e melhora na responsividade à insulina. Revista Brasileira de Fisiologia do Exercício. v. 10, n. 2, p. 107-109, 2011.

SANDS, A.L; LEIDY, H.J; HAMAKER, B.R; MAGUIRE, P; CAMPBELL, W.W. Consumption of the slow-digesting waxy maize starch leads to blunted plasma glucose and insulin response but does not influence energy expenditure or appetite in humans. Department of Foods and Nutrition, Purdue University. USA, 2009.

SAPATA, K.B; FAYH, A.P.T; OLIVEIRA, A.R. Efeitos do consumo prévio de carboidratos sobre a resposta glicêmica e desempenho. Revista Brasileira de Medicina do Esporte. v. 12, n. 4, p. 190-193 , 2006.

THOMAS, D.E; BROTHERHOOD, J.R; MILLER, B. Plasma glucose levels after prolonged strenuous exercise correlate inversely with glycemic response to food consumed before exercise. Internetional Journal of Sport Nutrition, v. 4, p. 361-373, 2006.

VOET, D; VOET, J. Bioquímica. 4. ed. Porto Alegre: Artmed, 2013. ; PRATT, C.W. Fundamentos de bioquímica. 4ª ed. Porto Alegre: Artmed, 2014.

WAITZBERG, D.L. Nutrição oral, enteral e parenteral na prática clínica. 4. ed. São Paulo: Atheneu, 2009.

WEBER, F.H; COLLARES-QUEIROZ, F.P; CHANG, Y.K. Caracterização físico-química, reológica, morfológica e térmica dos amidos de milho normal, ceroso e com alto teor de amilose. Ciência e Tecnologia dos Alimentos, v. 29, n. 4, p. 748-753, 2009.

ZHANG, G; VENKATACHALAM, M; HAMAKER, BR. Slow digestion property of native cereal starches. Biomacromolecules. v. 7, n. 11, p. 3252-3258, 2006. 